Article

\title{
From Attitude Change to Behaviour Change: Institutional Mediators of Education for Sustainable Development Effectiveness
}

\author{
Ismael Velasco ${ }^{1,2,3}$ and Marie K Harder ${ }^{1,2, *}$
}

1 Department of Environmental Science and Engineering, Fudan University, Shanghai 200433, China; E-Mail: Ismael@adorafoundation.org

2 Values and Sustainability Research Group, University of Brighton, Cockcroft Building, Lewes Road, Brighton BN2 4GJ, UK

3 Adora Foundation, Bristol BS5 8DA, UK

* Author to whom correspondence should be addressed; E-Mail: M.K.Harder@brighton.ac.uk; Tel.: +44-1273-600-900.

External Editor: Marc A. Rosen

Received: 18 April 2014; in revised form: 10 September 2014 / Accepted: 17 September 2014 / Published: 29 September 2014

\begin{abstract}
In this paper, we explore the way in which institutional contexts mediate values-focused behaviour change, with potential design implications. We use concepts taken from training research, where "learning transfer" refers to the translation into practice of the learning acquired during training: it is considered necessary to generalize it for the job context and for it to be maintained over a period of time on the job. In this paper, we analyse the example of one education for sustainable development (ESD) intervention that is already established as pedagogically effective when it is deployed in diverse institutional environments worldwide - the Youth as Agents of Behaviour Change program of the International Federation of Red Cross and Red Crescent Societies (IFRC). This allows an opportunity to consider variations in learning transfer due to distinctive moderating institutional features, which can now be understood in terms of varying transfer climates, levels of leadership support and opportunities to practice. Additional barriers of tokenistic consultation, lack of role clarity and perverse effects of increased distance between trainees and their colleagues on return were also seen. ESD programs intending to bridge the values-action gap could benefit from not focusing only on the training content, but pre-planning organisational support for returning trainees and including in the training ways for them to assess and plan to overcome such difficulties.
\end{abstract}


Keywords: behaviour change; institutional context; learning transfer; sustainable development; education for sustainable development

\section{Introduction: The Institutional Dimension of Education for Sustainable Development}

The goal of education for sustainable development (ESD), as defined by the United Nations Decade of Education for Sustainable Development (UNDESD), is "to integrate the principles, values, and practices of sustainable development into all aspects of education and learning. This educational effort will encourage changes in behaviour that will create a more sustainable future in terms of environmental integrity, economic viability, and a just society for present and future generations" [1].

At the heart of this perspective is the assumption or understanding that values and attitudes can shape behaviour. Once one can produce a shift in values and attitudes, it is held, the chances of changes in lifestyle will naturally grow. There is indeed evidence for this assertion, not least within the environmental arena [2-6]. Increasing voices have further suggested that to succeed in generating such urgent transformations, we need to consciously and systematically incorporate a deeper, at once more internal and more contextual dimension, designated variously as cultural-aesthetic, political-institutional or religious-spiritual [7]; or as we most recently argued, as "the missing pillar" of "ethical values" [8].

At the same time, Arbuthnott cautions against over-relying on a simplistic attitudes-behaviour relationship. Her research identifies that changes of intention, by themselves, are a variable and fragile mechanism for behaviour change, mediated by a range of non-attitudinal factors and most particularly contextual factors. Additionally, within contextual factors, her prescription for future ESD efforts is principally institutional [2] (p. 162).

However, this dictum does not resolve the issue of how ESD might best generate the behaviour changes in those putative leaders that would be needed for them to implement her prescriptions. It merely reinforces the need for successful ESD interventions that can change values, attitudes and behaviours, to be directed at such decision makers. She does however make clear that any such efforts need to be not only pedagogically sound, to the extent of succeeding in changing attitudes and values, but they need to be contextually embedded in "institutional infrastructures, regulations, and incentives" that can amplify and empower the otherwise weak effects of intentions on behaviour.

Not that the institutional dimension has been absent from the established conceptualizations of ESD. On the contrary, unlike most educational initiatives, the very origins of ESD do not stem from educational communities, but from institutional commitments from governments in the context of principally UN summits and their outcomes, of which UNDESD is but the most recent. As Hopkins and Mckeown encapsulate it, "ESD was crystallized when world leaders agreed that the concept of sustainable development should be actively pursued as a global goal" [9] (p. 14). He traces this to Rio 1992 and the launch of Agenda 21.

Thus, institutional processes and perspectives have been bound with ESD since its origins. It is only relatively recently, however, that the importance of institutional frameworks and structures has gained prominence as an intrinsic part of ESD initiatives in their own right. This is what the System of Environmental and Economic Accounting (SEEA) calls the "fourth-institutional-pillar" [10] (p. 5), 
and has been largely understood in terms of how ESD can influence the institutional context to make it and the communities those institutions serve more sustainable. The mediating role of institutional contexts on the intrinsic effectiveness of ESD interventions themselves, however, appears to remain largely to be explored.

In this paper, we draw on the "learning transfer" literature in the psychology of training, as a promising generative perspective from which to explore the way in which institutional contexts mediate values-focused behaviour change, not just descriptively, but with potential design implications. In training research, "transfer" refers to the translation into practice of the learning acquired during training. It specifies that for transfer to take place "learned behaviour must be generalized to the job context and maintained over a period of time on the job" [11] (p. 63). This literature was searched for proposed factors that are considered to mediate learning transfer. Those factors were used as a lens to consider variations found in the empirical data.

To explore those mediating factors that can help or inhibit, in this case, the learning about "the principles, values, and practices of sustainable development" from being transferred into practice, it was necessary to identify an ESD intervention that was known to be pedagogically effective as an agent of values and attitude change and which had been deployed in sufficiently diverse institutional environments to display variations in learning transfer that could be linked to distinctive moderating institutional features.

The Youth as Agents of Behaviour Change program of the International Federation of Red Cross and Red Crescent Societies was identified as such an intervention. A very detailed impact study of the first five years of the program [12] conclusively established that the training intervention achieved values and attitude change in almost all trainees, thus establishing the effectiveness of the program, even with different cohorts of trainees and different trainers. Yet, the degree of actual behaviour change, while very widespread, varied considerably in individual intensity and depth. In this paper, we interrogate the multiple datasets collected for that extensive impact study in light of the proposed mediating factors found in the learning transfer literature to posit key institutional mediators of behaviour change in ESD and suggest implications for the practice and research agenda of ESD post UNDESD.

\section{The "Youth as Agents of Behaviour Change" (YABC) Program: Establishing Effective Values and Attitude Change}

Although not positioned specifically as an ESD initiative, the Youth as Agents of Behaviour Change (YABC) program developed by the Department for Principles and Values of the International Federation of Red Cross and Red Crescent Societies (IFRC) is entirely centred on a proposed missing pillar of sustainability discourses, namely the sphere of values, missing particularly at the level of measurement and tangibility via clear indicators [8].

Concretely, the aim of YABC is to generate personal transformation and alignment with values through the acquisition of values-based skills to achieve consonance with the dictum "be the change you want to see". It seeks to prepare young people in the Red Cross/Red Crescent (RCRC) movement to become role models who can trigger in others the positive changes they have undergone themselves. 
Its strategy for preparing them consists of generating the commitment and motivation to self-transform and practically equipping them with peer education techniques and tools to mobilize others.

Its pedagogical methodology consists of generating affective [13] and transformational learning [30] through the generation of shared experiential self-discovery, using emotionally powerful non-cognitive methods (arts, games, role-play and Qi Gong, etc.). It involves an intensive 7-10 day training period focused on eliciting subjective experiences of values, upon which critical reflection first deconstructs and then rebuilds a personal identity around values, attitudes and intentions, with the aim of triggering lasting and contagious ethical behaviour change. The 75 exercises in its training and peer education toolkit have been co-designed and tested with youth in 107 countries.

In the case of YABC, the "transfer context" in which its ESD intervention is to be turned into practice is much wider than the bounded "job" arena that most of the transfer related studies have concentrated on and includes both their personal day-to-day lives and their RCRC work, whether as volunteers, staff or both.

In the context of $Y A B C$, effective transfer requires that $\mathrm{YABC}$ trainees:

- assimilate and exemplify the Red Cross/Red Crescent fundamental principles and humanitarian values;

- practice the YABC behavioural skills in their daily life;

- motivate others to replicate their change through role-modelling in their personal relationships and through application of the YABC toolkit and peer education techniques in the context of RCRC programs and their own social mobilization initiatives.

It is interesting to compare these with UNESCO's ESD Policy Dialogue 2 statement that the learning goals of ESD should couple "knowledge" to what is termed "action competence" and be designed to create "decision making capacity" in learners [14] (p. 3).

This "action competence" is said to integrate four elements, which together amount to "decision making capacity":

- Knowledge;

- Commitment and values;

- An interest in the future;

- Social, critical and creative thinking skills;

- Experience of real life situations.

These five elements can be said to align extremely closely with the pedagogical framework of YABC [15]. The implicit assumption in UNESCO's articulation of these foundational learning goals and components of ESD is that the creation of such action competence and decision making capacity will ensure the transfer of learning into behaviour congruent with and contributory toward sustainable development. Although it evolved separately and without these specific goals in mind, the YABC approach is so similar that it can be said to be an excellent example of such prime ESD pedagogy.

The recent independent impact study of its first five years concluded that the YABC conceptual framework, methodology and sustained process of co-design, testing and application in diverse national and identity contexts had resulted in an exceptionally powerful mechanism for effective values-, attitude- and behaviour-change, with documented impacts across countries and cultures, 
timescales and program settings [12]. The study concluded that the YABC pedagogy was remarkably effective, even across the range of trainee cohorts and trainers of different cultures. As noted earlier, however, there were variations in the extent of this effectiveness in different post-training contexts, suggesting that contextual factors moderated these effects. The work reported in this paper seeks to establish the role that institutional contexts specifically played in moderating the proven pedagogical effectiveness of the intervention.

\section{Research Methods}

(i) Literature review: to identify key findings in the literature on effective learning transfer, it was decided to build on the findings of existing systematic reviews. A keyword search was conducted on Google scholar using the terms "transfer training review" and "learning transfer review". Eleven systematic reviews were identified. Given that the aim of this study was not to produce a further systematic review, but to test whether salient factors identified in the literature were replicated in a successful ESD values and behaviour change intervention, it was decided to focus on the two most cited and hence most impactful reviews across two decades, namely Baldwin et al. (1988), with 2331 citations, and Burke et al. (2007), with 440 citations. Key references in these reviews were subsequently consulted first hand.

(ii) Secondary analysis of datasets used in the IFRC impact study [12] is detailed in Table 1 below. These include datasets from ongoing work of the IFRC, a joint study with a university as part of a bigger project [16] and primary data collected, specifically. While the strength of individual data sources was variable, the triangulatory robustness of consensus patterns across methods was strong, and in this paper, we use those findings that are confirmed by several methodologies or contrasting demographics, supported by illustrative, rather than typical examples.

This data was interrogated to find evidence of the presence or absence of the key learning transfer factors identified in the literature review and to note any newly revealed factors. Every dataset was examined for each factor, but some proved more valuable than others in each case due to their different characteristics, e.g., Global Online Survey for worldwide patterns, but the semi-structured interviews for in-depth local information.

Table 1. Overview of Youth as Agents of Behaviour Change (YABC) impact study datasets.

\begin{tabular}{|c|c|c|c|}
\hline Dataset & Key Questions/Issues Covered & Methodology & Robustness \\
\hline $\begin{array}{l}\text { Global Online } \\
\text { Survey (GOS) }\end{array}$ & $\begin{array}{l}\text { Perceived personal, social and professional } \\
\text { impacts on trainees, peer educators, and their } \\
\text { organisational setting over the medium and } \\
\text { longer term. }\end{array}$ & $\begin{array}{l}\text { Survey questionnaire } \\
\text { delivered online via } \\
\text { survey monkey }\end{array}$ & $\begin{array}{l}\text { Low to } \\
\text { Moderate }\end{array}$ \\
\hline $\begin{array}{l}\text { Internal } \\
\text { semi-structured } \\
\text { interviews (ISSI) }\end{array}$ & $\begin{array}{l}\text { Subjective impacts on trainees, peer educators } \\
\text { and their immediate environment over the } \\
\text { medium and longer term. }\end{array}$ & $\begin{array}{l}\text { In depth interviews } \\
\text { conducted by RCRC } \\
\text { staff mostly on Skype, } \\
\text { sometimes in person }\end{array}$ & High \\
\hline $\begin{array}{l}\text { Key informant email } \\
\text { questionnaires (KIQ) }\end{array}$ & $\begin{array}{l}\text { Observed impacts of YABC on trainees, peer } \\
\text { educators and their organisational contexts. }\end{array}$ & $\begin{array}{l}\text { email questionnaire } \\
\text { comprising } 2 \text { open } \\
\text { ended questions }\end{array}$ & Low to High \\
\hline
\end{tabular}


Table 1. Cont.

\begin{tabular}{|c|c|c|c|}
\hline Dataset & Key Questions/Issues Covered & Methodology & Robustness \\
\hline $\begin{array}{l}\text { Training evaluations } \\
\text { and field reports (FR) }\end{array}$ & $\begin{array}{l}\text { Self-assessed gains in knowledge and } \\
\text { understanding of the Fundamental } \\
\text { Principles and of YABC pre/ } \\
\text { post-training. Various aspects of training } \\
\text { event quality. Thematic areas of YABC } \\
\text { application in RCRC programmes. }\end{array}$ & $\begin{array}{l}\text { Pre and post-event } \\
\text { self-assessment } \\
\text { questionnaires and } \\
\text { event evaluation }\end{array}$ & Moderate \\
\hline $\begin{array}{l}\text { Brighton University } \\
\text { Independent YABC } \\
\text { Study (BTON) }\end{array}$ & $\begin{array}{l}\text { Values vocabulary of YABC trainees. } \\
\text { Behaviour impacts and impact } \\
\text { mechanisms. Cross cultural attitudinal } \\
\text { and behavioural priorities. }\end{array}$ & $\begin{array}{l}\text { Mixed methods research: } \\
\text { surveys, focus group, } \\
\text { semi-structured interviews, } \\
\text { non-participant } \\
\text { observation, scenario } \\
\text { analysis, vocabulary } \\
\text { analysis }\end{array}$ & High \\
\hline $\begin{array}{l}\text { Document } \\
\text { analysis (DOC) }\end{array}$ & $\begin{array}{l}\text { History, conceptual framework and } \\
\text { methodology of YABC. Implementation } \\
\text { of YABC. Contextual RCRC strategies } \\
\text { and programmes. Supporting academic } \\
\text { references on methodology. }\end{array}$ & Various & High \\
\hline
\end{tabular}

\section{Literature-Derived Institutional Factors of Learning Transfer}

\subsection{Institutional Enabling Factors of Learning Transfer and Behaviour Change}

Five key factors have been found in broader empirical research in the literature to enable behaviour change by learners in their organizational context post-training: transfer climate, strategic fit, opportunity to perform; accountability; and above all, peer and supervisor support.

\subsubsection{Transfer Climate}

Transfer climate refers to the prevalence of factors within an organization that either enable or obstruct the ability of trainees to transfer their learning into their work sphere, in this case, including voluntary work. The literature notes a number of key enablers of behaviour change in trainees' organizational contexts. Organisations that had a high appreciation for innovation, encouraged risk taking and allowed freedom to set goals were mentioned [17], as were those which favoured participation by subordinates, innovative behaviour and independence of thought [18]. Two other papers $[19,20]$ emphasized organisations with a culture of continuous learning.

\subsubsection{Strategic Fit}

Evidence has been presented to suggest that good strategic fit increases the likelihood that learning would be transferred by the learners to their organizational arena [21-23]. This was the case where there was alignment between the training received and the strategic direction of the organization, the trainees' learning outcomes matched the departmental goal, and the local decisions 
and daily work of the learners in their organizational context were linked by the organization directly to its organizational mission.

\subsubsection{Opportunity to Practice}

Opportunity to use trained skills was found to be a critical component of trainee skill maintenance in $[11,24]$ and was found to be the highest rated form of support in one study [22].

\subsubsection{Accountability}

Accountability is defined as "the degree to which the organization, culture, and/or management expects learners to use trained knowledge and skills on the job and holds them responsible for doing so" [13]. Accountability can signal to trainees the importance of their training to the organization [25]. Improved learning transfer was reported [26] where the sense of responsibility of trainees to implement their learning was increased by requiring post-training reports from them [26]. The act of informing of trainees that there would be post-training follow-ups or assessments likewise increased trainee learning transfer post-training [27].

\subsubsection{Supervisor and Peer Support}

This is by far the most important enabler of training transfer to the organizational context and the most amply evidenced in the literature [28]. Key management practices that greatly enhance the trainees' incorporation of new learning into their organizational practice include discussing new learning both pre- and post-training; helping trainees set training goals; participating in training and providing encouragement and coaching to trainees about use of new knowledge and skills on the job [19,29-32]. Trainees with more supportive supervisors entered training with stronger beliefs that training would be useful [27].

\subsection{Institutional Blocks to Learning Transfer and Behaviour Change}

The literature was searched for not only enabling but also blocking factors for learning transfer. Only three were found, as discussed below.

\subsubsection{Poor Transfer Climate}

Factors that have been identified as key constraints on the chances of post-training behaviour change include: shortages of or inappropriate resources; a lack of required services from others; a poor physical work environment; a lack of role relevant authority; work overload and crisis work [13,32].

\subsubsection{Low Opportunity to Practice}

There is evidence that, upon completion of training, trainees tend to face differential opportunities to practice and apply what they have learned. If their opportunities to practice are low or absent, they are likely to become de-skilled over time. Indeed research has consistently shown that positive transfer is limited when trainees are not provided with opportunities to use new learning [33-35]. One study 
suggested that limited opportunity to perform skills was the highest impediment to successful training transfer [36].

\subsubsection{Low Supervisor and Peer Support}

Lack of peer and specially supervisor support is among the most important blocks of post-training behaviour change. Among the related behaviours that have been demonstrated to obstruct learning transfer are a follow-up procedures with their returning trainees [37]; a lack of reinforcement or coaching or modelling [38]; a lack of manager support post-training even limited the positive influence of peer support on continued skill maintenance [39]; a lack of support from peers after training [13]; and a failure to convince older workers had negative effects on post-training behaviour change [32].

\section{Analysis and Discussion of the YABC Program in terms of Institutional Moderating Factors of Behavioural Change}

\subsection{Institutional Enablers of Learning Transfer in the YABC Program}

In the sections below, we present our analysis of the data sets in Table 1 with respect to each type of enabling institutional moderating factor.

\subsubsection{Transfer Climate (Organizational)}

The global online survey (dataset: GOS) data indicated that $68 \%$ of respondents reported that they receive adequate support from the leadership of their National Society to implement YABC initiatives (see Table 2 below).

Table 2. Question 14: Do you receive adequate support from your National Society Leadership to implement YABC initiatives? (dataset: GOS).

\begin{tabular}{lccccc}
\hline & Total surveyed & Response rate & \% Yes & $\begin{array}{c}\text { \% Did not ask for } \\
\text { support }\end{array}$ & $\begin{array}{c}\text { Variance } \\
\text { \% pts }\end{array}$ \\
\hline Americas & 61 & $92 \%$ & $72 \%$ & $18 \%$ & $4 \%$ \\
Asia & 78 & $94 \%$ & $68 \%$ & $20 \%$ & $0 \%$ \\
Europe & 65 & $95 \%$ & $71 \%$ & $26 \%$ & $3 \%$ \\
Middle East & 46 & $87 \%$ & $63 \%$ & $19 \%$ & $-5 \%$ \\
Average for all & & & $68 \%$ & $20 \%$ & \\
\hline
\end{tabular}

A further $20 \%$ stated that they had not asked for support, and $12 \%$ stated that they had not received the support they needed.

As a global phenomenon, this indicates a good-to-excellent Red Cross/Red Crescent culture in terms of welcoming the dynamics that YABC brings to an organization, consistent across different global zones. This would suggest a generally positive global transfer climate for peer educators to integrate the new learning into their work. A healthy organizational transfer climate for YABC trainees is clearly frequent across the board.

However, it should be noted that the majority of YABC peer educators do not have strategic institutional roles, and any interface with their national society is at a more personal and comparatively 
superficial level. Thus, this data could be described as indicating perceived support at the individual level. When it comes to actual institutional enabling and engagement with YABC the picture is much more complex, as will be made clear in the Section 5.2 when looking at resistance to change under organizational impact blockages.

\subsubsection{Strategic Fit}

Seventy three percent of question respondents reported that YABC was now integrated into the strategic plans of their National Society, and $75 \%$ of question respondents said that YABC activity was integrated into mainstream programs, further suggesting trends of strategic fit at a more immediate, local organizational level (dataset: Global Online Survey (GOS)). The YABC originated in the global office of the IFRC, in the IFRC Principles and Values Department, which is involved in the high level formulation of international (not national) organizational strategies (dataset: document analysis (DOC)). Thus, the YABC's initiation, strategic ownership and championship from that influential global strategic management level implies a strategic fit starting at the top and then cascading down the (autonomous) national societies that make up the federation (IFRC).

A national origin and design for YABC might not have been globally transferrable in terms of strategic fit and, therefore, less effective in leveraging support and investment by other National Societies. Conversely, Section 5.2 shows difficulties arising from incomplete top-down cascading.

\subsubsection{Opportunity to Practice}

No matter how dedicated the peer educators were post-training, if the organization did not create or allow opportunities for the peer educators to conduct YABC-inspired activities then the impact of YABC, both behavioural and institutional, would have been dramatically less.

Table 3. Pattern of social engagement by peer educators and trainees (dataset: GOS).

\begin{tabular}{ccc}
\hline Social Engagement Activity & $\begin{array}{c}\text { No. Peer educators } \\
\text { Involved }\end{array}$ & \% (of 185 respondents) \\
\hline $\begin{array}{c}\text { Presentations of the YABC initiative } \\
\text { (duration 0 to 4 h) }\end{array}$ & 114 & $73 \%$ \\
\hline $\begin{array}{c}\text { Workshops, sessions or activities using the YABC toolkit } \\
\text { (duration 4 to 12 h) }\end{array}$ & 77 & $78 \%$ \\
\hline $\begin{array}{c}\text { Workshops, sessions or activities using the YABC toolkit } \\
\text { (duration over 12 h) }\end{array}$ & 79 & $49 \%$ \\
\hline $\begin{array}{c}\text { Social mobilization/awareness-raising campaigns using } \\
\text { creative platforms on YABC-related topics }\end{array}$ & 77 & $50 \%$ \\
\hline $\begin{array}{c}\text { Community-based micro-projects on } \\
\text { YABC-related topics }\end{array}$ & 64 & $49 \%$ \\
\hline Other (if any) & 35 & $22 \%$ \\
\hline
\end{tabular}

The data showed that a very high proportion of participants ( $c a .70 \%)$ spontaneously initiated their own follow-up activities post-training, with an average of 37 YABC-related spontaneous YABC 
activities per participant (See Table 3: dataset: GOS). The averages are not representative, as some will have engaged in literally hundreds of activities while other individuals might undertake a single one. What is conclusive, however, is the generally typical scope of opportunities to practice, which given the research literature, is therefore likely to have been a critical factor in YABC's success in generating behaviour change.

\subsubsection{Accountability}

The data provides little evidence for the presence or absence of specific accountability mechanisms, although several trainees or peer educators who are also in leadership or coordination positions mention having to write reports, demonstrate impact and justify investment (dataset: internal semi-structured interviews (ISSI), key informant email questionnaires (KIQ), Brighton University Independent YABC Study (BTON), Training evaluations and field reports (FR)). The vast majority of trainees, who are not in strategic leadership positions, would not have this mechanism in place.

However, the data pointed to a degree of informal "soft" accountability generated by the IFRC's Principles and Values Department, whose YABC global project coordinator carried out very active follow-up, encouragement and contact with trainees. The interview data documenting her perception of trends, overall, by geographical zone, and with identifiable individuals, was positively triangulated with data emerging from other datasets (datasets: KIQ, GOS, FR), which indicated that trainees are reminded and encouraged through regular written communications, frequently contacted by Skype, connected through online social networks, and often met in the course of successive training events across the world.

Such links from individual trainees to a department located in the global IFRC office in Geneva, and to fellow YABC trainees worldwide, would of themselves clearly signal the global institutional importance of their local and individual efforts and, thus, incubate a sense of responsibility and accountability.

\subsubsection{Peer Support}

As mentioned in the sub-section above, a strong level of peer support worldwide can be inferred from the data (datasets: KIQ, GOS, FR) showing trainees are connected through social networks, subsequent training events and informal networks set up through the office in Geneva. However, peer support can also be said to be present in the peers engaged on the return of the trainee to his workplace, as the YABC program is focused on peer education. Proxy indicators from the global online survey indicated that an estimated 120,000 peers were engaged by the trainees. While the number reached by each trainee varied greatly, virtually the entirety of the sample reported some peer engagement.

\subsubsection{Supervisor Support}

The high levels of reported satisfaction by trainees with the levels of institutional support that they received is a strong indication of adequate supervisor support (dataset: GOS).

In addition, the following proxy indicators may be said to corroborate such a conclusion (GOS, ISSI): 
- the general incorporation of YABC initiatives or tools into existing national programs;

- the integration into a substantial number of national strategic plans;

- the plans by a significant number of national societies for translation of the materials into local languages, requiring human and economic investment by the organization.

As all of these indicators would mostly or wholly require hierarchical approval, they may be considered strong indicators of generally good levels of supervisor support. An example of this is provided by a $\mathrm{YABC}$ trainer peer educator:

"The strength of YABC is that you can integrate it into different areas, sensitize more people and mobilize them. For example, the managers of the blood donor programs, they are the ones who want to integrate it and ask me to shape the module. Managers are present during the youth camps so they can see what is $\mathrm{YABC}$, and they know everything about the YABC program. Furthermore, by the end of every youth camp, the youth members present a declaration and resolutions and request the senior management to integrate the YABC in all activities and trainings." (Trainer peer educator, South East Asia).

\subsection{Organizational Blocks of Behaviour Change}

In contrast to the pedagogical elements of the YABC training, where the feedback has been almost uniformly positive, when it comes to organizational blocks to learning transfer and the deployment of YABC concepts more generally, the data, from both trainees and external strategic observers, documents the presence of significant obstacles and barriers from local to international level (dataset: ISSI, KIQ, BTON).

It is important to emphasise that these were either comparatively rare or rather specific, and the barriers explored below are not typical of the majority. However, they are worthy of closer study, because they are instances of the type of institutional barriers that can be encountered in the translation from training into behaviour and were, in aggregate, reported by significant minority of participants in this study.

To contextualize this, we can recall that in the global survey more than one in 10 (12\%) of trainees stated explicitly that they do not feel adequately supported by their National Society (dataset: GOS). Likewise in the Jordan study of a cohort of international trainees who had already achieved 1-2 years of intense, sustained and institutionally engaged YABC activity in their countries, they described typically facing significant and ongoing organisational barriers (dataset: BTON).

Thus, while we can say that on the whole the organizational climate of the RCRC has been nurturing and healthy for the deployment of YABC over its first five years, it can also be said that the presence of organizational blockages is significant enough that it is reasonable to expect that the full potential of YABC impacts is not being released.

The factors found to be important in this study are consistent with those mentioned in the literature (Section 4.2), but others were also reported:

- Tokenistic consultation/superficial institutional engagement;

- Lack of role/progression clarity;

- Increased distance between personal values and institutional culture; 
- In-group/out-group dynamics.

These factors are not typical of the majority, who stated that they feel supported or do not need support, ando no one factor necessarily represents the experience of others within the $12 \%$ who stated they felt institutionally blocked. They may be seen as negative case samples that may illustrate the range and types of challenges that trainees may sometimes face, even if the specific challenges will vary.

\subsubsection{Poor Transfer Climate}

Our data revealed evidence of the resources factor suggested by the literature (Section 4.1.2), and also revealed unexpectedly rich detail on the factor of "lack of required services from others": both are discussed below. No clear evidence was found for work overload, crisis work or poor physical work environment, but the factor of "a lack of role-relevant authority" overlaps with several other factors in our data.

\subsubsection{Resource Barriers}

Funding difficulties for new, YABC-related programs were noted by a number of respondents (datasets: ISSI, KIQ, BTON). In one case, the trainee commented that finding sufficient funding was the major challenge in implementing YABC training:

"Funding is always a problem, for example if I want to organize two trainings next year then I don't have the funds for it. Right now I am starting to think about partnerships to get the funding, because their focus is also youth", Trainee, South East Asia, Male

The challenges of administration and the logistics of mobilizing and coordinating young people to implement YABC-inspired initiatives was also noted. Although reported as a resource-related problem, it could be seen as more akin to the marginalisation factor reported in Section 5.2.1.2b: marginalised programs are less likely to be funded.

\subsubsection{Lack of Required Services from Others: Resistance to Change}

An organizational culture that resists change was noted in some localities resulting in resistance to the YABC training program itself. This manifested in a number of ways.

Fixed agendas: In the Jordan study by Brighton University, the researchers carried out an exercise called scenario generation and analysis (dataset: BTON). Trainees were asked to share a personal experience that illustrated examples of a time where they felt very empowered to make a change and an experience where they felt very disempowered to do so as YABC trainees. A Likert scale for empowerment was then created, with points associated to elements in the story. The remaining trainees were then asked whether the anecdotes shared were recognisable to them as good examples of those two Likert scale poles in their own experience, even though the anecdote was someone else's. Once everyone agreed that the scale was valid for them and which gave measures of empowerment valid in their own experience, they were asked to place a circle in the point in the spectrum which was most typical of their day to day experience as YABC peer educators, from very rare to very typical. 
In this case, five out of six trainees who participated in the scenario analysis, some two years into their very active service in their national societies as YABC peer educators, considered it was extremely typical for them to have to struggle in the face of fixed institutional agendas.

This result for international level trainer-peer educators differs greatly to the $68 \%$ result of feeling supported registered for non-trainer peer educators, shown in Table 2 and Section 4.1.1 and could be an indication of various things. These trainees would have had more exposure to and awareness of national level agendas or those could have been more inflexible than local agendas where most of the other trainees operated. That the international trainees in that sub-study came from four different global zones suggests a widespread IFRC issue. An interesting finding that comes from triangulation of the Jordan study with the global online survey data is that a number of those reporting typical institutional resistance to YABC, due to fixed agendas, were also among the most active and highperforming trainees in terms of the behaviour changes they were achieving in projects. This underscores the possibility that some positive effects can mask a much greater potential that is being held back by organizational factors; the impact measures are of partial attainment, not of full actualisation.

(a) Marginalisation Due to Unfamiliarity and Complacency

A commonly reported source of resistance to change was a sense of complacency vis-à-vis the Fundamental Principles of the Red Cross/Red Crescent and a lack of familiarity with the distinctive features of YABC (dataset: ISSI, KIQ, BTON). Thus, one strategic observer described that

"a lot of people did not take part in YABC either because they did not see the usefulness of the program or because they considered that they already knew enough about the principles. It is good to highlight that the YABC approach is unknown to most people (volunteers mainly), which contributes to creating this resistance", Former Coordinator of Relief Operations during Civil Unrest.

As hinted in this quote, the YABC training methodology can be misjudged and seriously under-estimated, confusing the non-cognitive mechanisms with mere entertainment, to the degree that the YABC approach was marginalised against more traditional or familiar training methods:

"The first major problem as we started the program from the very beginning was that no one knew about YABC. It was very new for my NS and they took it very lightly, "what do you mean when you are playing games with 30 volunteers"; they thought it was more useful to train volunteers on first aid and other trainings." Trainee, Middle East, Male

This phenomenon is clearly not unusual, at least in the early stages of introducing YABC, as yet another trainee shares:

"The challenge was to convince the provincial branches about YABC program and its methodology. As the program managers were used to the traditional way of learning, they were following the traditional methodology; the YABC was totally a new concept. They were reluctant to change (there was resistance to change, not cultural problem). The program managers who were reluctant to change inform the Secretaries and say they don't want this program." Trainee, Male, South East Asia. 
All three of these testimonies are balanced by the fact that the interviewees in Jordan reported that they largely overcame the initial scepticism and barriers until YABC has become a core part of their National Society activity. However, it re-emphasises the emergent finding that successes as reported so far may be underestimates of potential.

\section{(b) Generational Gaps}

Resistance to change was also perceived around generational gaps in one region, impacting upon the potential for integration and replication of the YABC approach. It was suggested that whilst young people may readily accept new ideas and be prepared to make significant changes in their world views, this was more difficult for older generations.

"Bringing YABC to program managers and in general to non-youth in [this country] would be a challenging issue. I see it as very necessary, but it would be a very different piece of cake than bringing it to youth, what has been done so far. This said, it is not impossible: it's just that it's much easier to talk about values with youth than with senior people in this or in any country. Youth are usually much more open than others to change." IFRC Head of Delegation, Middle East.

\subsubsection{Limited Opportunities to Practice}

This manifested in two ways:

(a) Limited Structured Support for Participants to Implement Their Ideas

Trainees reported that opportunities to practice needed to improve beyond general possibilities available, to include structured support and accompaniment in turning attitudes and values change into behaviour and to turn ideas and intentions into practice (dataset: ISSI, KIQ):

"When you go back to your country after the training, a young person who has just been trained needs a section, a structure where they are included to carry out these activities. It is true we do it (use YABC) in our daily life, but in reality if we want to see a change, we need to be supported by the Red Cross, we need to be supported by the group we belong to. It is more difficult for people to conduct activities by themselves. It is unrealistic, because a young volunteer when he comes back from a training, needs to be included in a group or the [National Society] youth section. It is more realistic then for him to conduct activities." Trainee, Western Europe, Female.

This was a recurrent issue, and an important one. There are indications that a proportion of the trainees require greater support locally to complete the transfer of their learning into practice. One suggested that part of the YABC training should include guidance on how to integrate with the plans and structures of their national society, and have a clear bridge into a local support structure as suggested above, without which, it was strongly implied, learning transfer would greatly suffer, and practical application in relation to social mobilization might become, in the words of the previous citation, "unrealistic". 
"For example, in the RCRC YABC group on Facebook, there are many people from NS asking me what to do next. They are asking me who to contact in their National Society. So they don't have a clear vision of what they should do next. In the training, the last day we ask them to develop a plan for one year, but I think we should do more, we have to come up with something else. We (IFRC) should think more on how to link their plan with the NS and put it in the training pack. So we should include in the toolkit and the training some guidelines of what they can do and how, like options they can explore including next steps after the training, for example they can work in communities, they can do integration, etc.) and also how to link with the National Societies." Trainee, South East Asia, Male.

(b) Inadequate Program Integration

In the global online survey (GOS), 75\% of question respondents reported that the implementation of YABC was integrated into their National Societies programs. Although this sounds positive, it does mean that $25 \%$ have inadequate integration, and three other datasets indicated that this caused a significant barrier to learning transfer (dataset: ISSI, KIQ, BTON):

"One of the gaps is the lack of link with other programs, for example where there is no integration of $\mathrm{YABC}$, and if YABC is conducted on its own, then I think it is a gap. YABC should be included within the tools that a National Society uses to develop its strategic plan. You can train people and then what? If they don't integrate it into other work areas, then I think it is not effective." Trainee, Western Europe, Female.

This evidence is in line with findings in the learning transfer literature.

It was also noted that integration needs to be within long-term, sustained programs, and not activities with a short term focus only.

"To be sustainable YABC should be included in programs having a long term vision, like a 5 years program, we need to include it in programs which are not going to abandoned by the National Society, programs which are sustainable themselves." Trainee, Western Europe, Female.

\subsubsection{Low Leadership Support}

This was the third key barrier to behaviour change identified in the learning transfer literature, and in fact, the most commonly cited institutional barrier to the efficacy of the YABC program: lack of leadership support at the National Society Level. Trainees stated that YABC has not been highly regarded by many at the leadership level, creating obstacles to its implementation (dataset: ISSI, KIQ, BTON).

"There is no full acceptance from the leadership level, and that makes our process slower to get the desired results. Right now, what's happening is that it is the Federation pushing for it. For example, we have excellent trainees in this region but D was not authorized to go for the training in Sri Lanka. This is because of the lack of understanding of the whole concept of YABC at the National Society leadership level. They think it is just a training like any other." Trainee, South East Asia, Male. 
There are several aspects of this point which overlap with other factors we found: here we focus on examining in more detail on why the presence or absence of leadership support for the YABC trainees seemed to be such a key factor.

It was stressed by several respondents that until there is a strong sense of ownership of the program across strategic, operational and volunteer levels then the proper traction will not be achieved.

"One thing is that it should be institutionalized, and everyone should be aware and really understand the YACB concept, from governance and management side. The leadership, senior management and youth themselves should take the lead on this, but they can take the lead only when they understand the importance of it. There should be a clear acceptance from the leadership. How can we reach to this? I think we should ask the leaders of the NS to get the solutions." Trainee, South East Asia, Male

The consensus of the qualitative data, from both internal and independent observers, was that the principal factor that tips the RCRC leadership away from the support required was simple lack of familiarity. Some participants have, however, found that this can be overcome where managers are able to observe the impact of the YABC program in practice and are won over to the approach.

"In the beginning it was very difficult, and bit by bit it was accepted. Now it is very popular. when they talk about youth they talk about YABC, when they talk about training they talk about YABC. We have personal relationships with project managers and we explained them the benefits of $Y A B C$, then we conducted the trainings, and with the evaluations we got from participants, we could show that to the project managers for them to believe. Then they are the ones convincing the senior management." Trainee, Middle East, Male.

Further, where training takes place at a national, rather than international level, it may be more likely that senior and program managers will take part, increasing their exposure to and confidence in the approach.

"I think it is important that National Societies know about program and all yes, but at the same time I think it is important that there is a large number of volunteers from the same National Society to be able to start together. When it is a national training it is always better, because maybe the Head is participating, more people from the National Society participating, and also it is important to have both volunteers and staff during the training of peer educators." Trainee, Western Europe, Female.

\subsubsection{Additional Barriers}

While the institutional barriers reviewed above align closely with those mentioned in the existing literature on learning transfer, our study also found evidence for additional institutional factors that were perceived by trainees to obstruct their capacity to fully implement their attitudinal and values changes and their concomitant intentions to act, into fully effective behavioural practice.

(a) Tokenistic Consultation/ Superficial Institutional Engagement

A challenge encountered sometimes is what a trainee in Jordan referred to as "false consultation", meaning tokenistic gestures of creating spaces where youth in the society can articulate their ideas and 
priorities, and even get nominal acceptance and effusive encouragement, yet not translate into significant influence on decisions or on outcomes. This appears to be a significant problem, as nine out of nine trainees in Jordan who participated in this scenario analysis, from seven national societies and five global zones unanimously experienced this as typical in their experience since they first trained (dataset: BTON).

\section{(b) Lack of Role/Progression Clarity}

One of the areas that remained blurred at the time of the study was the exact point at which a peer educator becomes a trainee (dataset: ISSI, KIQ, BTON).

"Another problem is that nobody understands the process of becoming a trainee. We don't know the official line for Peer Educators who have been coached. Nobody knows when you are an official trainee, there is no clear guidance. We don't know if she (a trained participant) is allowed to train others in [that country] now. Giving coaching for Peer Educators is ok, but we don't know if now can go ahead you are a trainer "officially." Trainer, Western Europe, Female.

It is worth bearing in mind this comment is coming from one of the experienced international trainers. This a critical constraint, as in a number of areas the expansion of the YABC program is stalled for lack of training capacity.

\subsubsection{Perverse Effects}

Perverse effects are a type of unintended consequence, where successful implementation leads to results that subvert the intended outcomes. Although these were very rare in the data, they provide proof of concept that indicate more generic risks. These perverse effects were born from of the interaction between a highly successful values, attitude and behaviour change ESD intervention, and an institutional context in tension with those positive results. As such, they might be considered more generic risk factors to be taken into account more generally in learning transfer and ESD programs.

(a) Increased Distance between Personal Values and Institutional Culture

The very effectiveness of the YABC program occasionally generated perverse effects where the distance between trainees' newly developed values and attitudes and those of their colleagues and peers within their institutional environment was too high upon their return from training. This produced cognitive dissonance in some of the trainees, as they felt the distance between institutionally espoused values and institutional practice with new acuteness, finding it more challenging. Where the institutional support mechanisms were not in place, the resulting cultural distance could in fact prove de-motivating.

In one case (out of 270 survey participants), a trainee reported a seriously dysfunctional institutional context in her locality, which removed all possibility of learning transfer within the organization and produced disenchantment with the Red Cross Red Crescent as a whole. She had found this challenging before the training, but post-training it became intolerable (datasets: GOS, KIQ).

While this is an extreme and atypical case in the data, it may be considered the far end of a spectrum of cognitive dissonance that can be produced by effective ethical transformation in an ESD 
intervention followed by return to an institutional environment perceived as ethically incongruent, and similar effects do emerge from the data in milder forms.

(b) In-Group/Out-Group Dynamics

A further occasional perverse effect was that the close cohesion of cohorts of YABC trainees could make peers and colleagues within their Red Cross/Red Crescent institutional context feel a disconnect from the newly bonded trainees (dataset: ISSI). On one occasion youth in the organization who had not been selected for the training felt excluded and thus diminished their engagement with the peer educators, inhibiting the trainees' learning transfer and creating an unwelcoming climate. Although rare, this is illustrative of a spectrum of response that is likely to be more or less present depending on the wider institutional context of trainees before and after training.

\section{Discussion}

This study has used knowledge from the learning transfer literature to explore organisational enablers and barriers in an ESD program. The learning transfer concepts were found to be applicable and useful in this analysis, facilitating the identification and articulation of specific learning for the RCRC to take forward. Additional barriers were also identified, namely tokenistic consultation, lack of role clarity and perverse effects, such as increased distance between trainees and their colleagues at home, due to significant values shifts in them and feelings of exclusion by their colleagues. These findings suggest that the fields of ESD and learning transfer are useful to each other, and perhaps this link will develop further.

In articulating the goals of ESD in the context of the Millennium Development Goals (MDG) [14], UNESCO identified the knowledge-action gap as a key challenge identified in relation to multiple MDGs. In the context of this paper, this gap can also be described as a failure of "learning transfer", where individual knowledge held about sustainable development practice does not eventuate in actual personal behaviour. This study supports findings from the learning transfer literature in training studies indicating that there are contextual and, more precisely, institutional factors that constrain or liberate the individual's "decision making capacity" independent of personal attitudinal and values shifts and sincere intentions to change.

This may be one of the factors behind Webb and Sheeran's findings that "a medium-to-large sized change in intentions engenders only a small-to-medium change in behaviour" [40] (p. 262). They go on to state that "intentions have less impact on behaviour when participants lack control over the behaviour, when there is potential for social reaction, and when circumstances of the performance are conducive to habit formation." [40] (p. 262).

Clearly, institutional contexts can greatly influence, positively and negatively, the degree of control individuals have over given behaviours, the type and intensity of social reaction within organisational contexts, and the opportunities, incentives and spaces for habit formation.

Webb and Sheeran do not in fact explore this dimension, but their work is a starting point to Arbuthnott's recent questioning of the sufficiency of attitudinal and intentional change for achieving behaviour change, and concluding that: "changing the context to make environmentally sustainable behaviour easier and/or more rewarding will increase behaviour change regardless of one's attitudes. 
Thus, directing ESD efforts to decision makers who can change institutional infrastructures, regulations, and incentives such as financial benefits or social approval will have the greatest impact on sustainable behaviour" [2] (p. 162).

Arbuthnott, writing in 2008, suggests that her work is the first to relate to ESD the findings on the insufficiency of attitudinal and intentional change for behavioural change, and the power of institutional mechanisms and contexts to achieve behaviour change even absent values and attitude change. Her research however does not go a step further to identify in detail evidence-based institutional factors that moderate the behavioural impact of ESD specifically. Her recommendations extrapolating from the psychology of intention and behaviour do however emphasise the significance of targeting ESD at institutional leaders, and practice-focused pedagogy institutionally incorporated into university ESD programs (p. 160).

This paper introduces a new dimension or next step in understanding the institutional context of ESD effectiveness, by suggesting an evidence-based approach and outline of key organisational factors that may moderate the behavioural effectiveness of ESD training. Beyond a recognition of the importance of institutional factors in general, it complements and indeed supplements UNESCO's conception of "decision making capacity" as the end goal of ESD.

Where decision making capacity in UNESCO's articulation is atomised in the individual as an ESD learning outcome, this paper helps to delineate the contours of a limited and concrete set of institutional dimensions that the evidence shows may have the greatest impact on learning transfer, that is, the translation of pedagogically imparted knowledge, attitude and values change into actual behaviour. Its findings suggest that successful ESD interventions must go beyond pedagogy, and even beyond attitude and values change. That it is not enough to plan or indeed achieve excellence in the ESD content, methodology, instructor recruitment and training cohorts. That for maximum impact, preparatory and follow up work in post training, real life organizational settings may dramatically enable impact, and that the absence of such institutional engineering, both "soft" (e.g., leadership buy-in and trainee support) and "hard" (e.g., accountability frameworks and opportunities to practice) would be problematic. This might indeed account for the relative ineffectiveness of the ESD Decade in significantly denting the values-action gap worldwide.

In our study, where effective post-ESD institutional contexts (with the greatest behavioural and institutional impacts) were found, they were often systematic, with conscious and methodical, if intuitive efforts to secure the core elements identified above. In the contexts where such enabling institutional conditions were not found, it was often owing to the lack of capacity in the trainee to generate such institutional processes upon return to their local work environment: rarely were the institutional conditions consciously and systematically in place ahead of the ESD training intervention.

\section{Conclusions}

Concepts of organisational enablers and barriers from the learning transfer literature were found to be significantly useful in identifying these in the context of ESD. The pedagogically successful RCRC YABC program had shown variations in learning transferred, which, with regard to institutional context, can now be understood in terms of varying transfer climates, levels of leadership support and 
opportunities to practice. Additional barriers of tokenistic consultation, lack of role clarity and perverse effects of increased distance between trainees and their colleagues on return were also seen.

ESD programs anxious to bridge the values-action gap could benefit by pre-planning organisational support for returning trainees and including in the training ways for them to assess the potential of and overcome such difficulties.

\section{Limitations and Recommendations for Future Research}

Limitations to the study include the fact that its methodology was neither a meta-analysis, nor a grounded theory approach, but rather a mostly retrospective analysis of secondary data collected for other purposes. As such, it is strictly speaking an exploratory study, and a more rigorous investigation of its findings should be undertaken before being taken as proven. Although the extent of the study across large numbers of trainees and cultures and home organisations provides some mitigation to this problem, it is still possible that important aspects of the learning transfer were intrinsically missed.

This paper proposes that an evidence-based approach to the planning-in of contextual and institutional integration into ESD training design and interventions, incorporating such vectors as have been discussed here, should be promoted.

To maximise behavioural transfer of values and attitude changes and intentions to act, advance institutional planning for such transfer is critical. This could take place in three stages:

(a) Diagnostics: a pre-assessment of the potential presence or absence of the enabling and obstructing institutional factors identified in this study could be made ranging from simple internal reflection by training organizers or commissioners or more formal and rigorous assessment and evaluation involving home organisations.

(b) Institutional planning and corrective action: on the basis of the above, to reinforce enabling institutional factors and address gaps ahead of the training intervention, and plan how the training will be followed up by appropriate institutional action and mechanisms, such as leadership involvement and support and opportunity to practice.

(c) Evaluation for organisational learning: to monitor and evaluate attitude and behaviour change and learning transfer immediately post training and again at a later period. This could then become the new baseline for any subsequent ESD interventions, and can also inform institutional development.

These three stages can take place with a spectrum of rigour dependent on institutional scale, context, goals, investment and resources. It can range from semi-formal reflection by a small group, through systematic action research, to significant strategic, professional and/or academic evaluation and planning.

Future areas of potentially fruitful research include exploring how such evidence-based institutional/organizational elements that maximize ESD transfer apply to different kinds and levels of institutions, from the grassroots to national and international guiding institutions.

It would be also be interesting to consider whether and how these findings and the transfer literature in general are applicable to ESD in formal education, as opposed to the training context considered here.

Finally, the focus on "transfer" mechanisms, i.e., enablers and barriers as a way of thinking of the link between intentions and behaviour, might be a potentially useful approach at the level of 
sustainability diplomacy, in terms of developing an evidence base for what kind of institutional engineering, soft and hard, might be put in place in state and inter-governmental institutions to maximize transfer from summit declarations, strategies and commitments into leadership behaviour, including, but not limited to, compliance.

\section{Acknowledgments}

The authors would like to acknowledge the support of the China National Thousand Talents Plan which supported this work.

\section{Author Contributions}

Ismael Velasco carried out the main design and performance of the research, anlayzed the data and wrote the first draft. Marie K Harder assisted with the design, analysis and synthesis, and made substantial contributions to the writing of the paper. Both authors read and approved the final manuscript.

\section{Conflicts of Interest}

The authors declare no conflict of interest.

\section{References}

1. United Nations Educational, Scientific, and Cultural Organization (UNESCO). Education for Sustainable Development. Available online: http://www.unesco.org/new/en/education/ themes/leading-the-international-agenda/education-for-sustainable-development/mission (accessed on 10 March 2014).

2. Arbuthnott, K. Education for sustainable development beyond attitude change. Int. J. Sust. Higher Educ. 2009, 10, 152-163.

3. Nickerson, R.S. Psychology and Environmental Change, Erlbaum; Psychology Press: Mahwah, NJ, USA, 2003.

4. Joireman, J.A.; van Lange, P.A.M.; van Vugt, M. Who cares about the environmental impact of cars? Those with an eye toward the future. Environ. Behav. 2004, 36, 187-206.

5. Stern, P.C. Toward a coherent theory of environmentally significant behaviour. J. Soc. Issues 2000, 56, 407-424.

6. Heath, Y.; Gifford, R. Free-market ideology and environmental degradation: The case of belief in global climate change. Env. Beh. 2006, 38, 48-71.

7. Littig, B.; Griessler, E. Social sustainability: A catchword between political pragmatism and social theory. Int. J. Sustain. Dev. 2005, 8, 65-79.

8. Burford, G.; Hoover, E.; Velasco, I.; Janoušková, S.; Jimenez, A.; Piggot, G.; Podger, D.; Harder, M. Bringing the "Missing Pillar" into Sustainable Development Goals: Towards Intersubjective Values-Based Indicators. Sustainability 2013, 5, 3035-3059. 
9. Hopkins, C.; Mckeown, R. Education for sustainable development: An international perspective. In Education and Sustainability: Responding to the Global Challenge; Tilbury, D., Stevenson, R.B., Fien, J., Schreuder, D., Eds.; Commission on Education and Communication: Gland, Switzerland; International Union for Conservation of Nature and Natural Resources (IUCN): Cambridge, UK, 2002.

10. European Commission: EUROSTAT. Available online: http://www.insee.fr/en/insee-statistiquepublique/colloques/acn/pdf10/ravets.pdf (accessed on 3 July 2013).

11. Baldwin, T.T; Ford, J.K. Transfer of Training: A Review and Directions for Future Research. Pers. Psychol. 1988, 41, 63-105.

12. Velasco, I. From the Heart to the Head: A 5 Year Impact Study of the Youth as Agents of behaviour Change (YABC) Program, 2008-2013; International Federation of Red Cross and Red Crescent Societies: Geneva, Switherland, 2014.

13. Burke, L.A.; Hutchins, H.M. Training Transfer: An Integrative Literature Review. Hum. Resour. Dev. Rev. 2007, 6, 263-296.

14. Education for Sustainable Development and the Millennium Development Goals. Available online: http://unesdoc.unesco.org/images/0017/001791/179120e.pdf (accessed on 9 September 2014).

15. Youth as Agents of Behavioural Change (YABC). Available online: http://www.mediterraneumrc.org/ pls/portal30/docs/PAGE/CANCRE/CCM/ISSUES/CCMYOTH/ATLANTISIXBOSHERZ/TAB4 2335537/THE\%20YABC\%20INITIATIVE.PDF (accessed on 21 August 2014).

16. Podger, D.; Piggot, G.; Zahradnik, M.; Janouskova, S.; Velasco, I.; Hak, T.; Dahl, A.; Jimenez, A.; Harder, M.K. The Earth Charter and the ESDinds Initiative: Developing Indicators and Assessment Tools for Civil Society Organizations to Examine the Values Dimensions of Sustainability Projects. J. Educ. Sustain. Dev. 2010, 4, 297-305.

17. Baumgartel, H.J.; Reynolds, J.I.; Pathan, R.Z. How Personality and Organizational Climate Variables Moderate the Effectiveness of Management Development Programs: A Review and Some Recent Research Findings. Manage. Labour St. 1984, 9, 1-16.

18. Hand, H.H.; Richards, M.D.; Slocum, J.W. Organizational Climate and the Effectiveness of a Human Relations Training Program. Acad. Manage. J. 1973, 16, 185-195.

19. Tannenbaum, S.I.; Smith-Jentsch, K.A.; Behson, S.J. Training Team Leaders to Facilitate Team Learning and Performance. In Making Decisions under Stress: Implications for Individual and Team Training; Cannon-Bowers, J.A., Salas, E., Eds.; American Psychological Association: Washington, DC, USA, 1998; pp. 247-270.

20. Tracey, J.B.; Tannenbaum, S.I.; Kavanagh, M.J. Applying Trained Skills on the Job: The Importance of the Work Environment. J. Appl. Psychol. 1995, 80, 239-252.

21. Montesino, M.U. Strategic Alignment of Training, Transfer-Enhancing behaviours, and Training Usage: A Posttraining Study. Hum. Resour. Manage. Quar. 2002, 13, 89-108.

22. Lim, D.H.; Johnson, S.D. Trainee Perceptions of Factors That Influence Learning Transfer. Int. J. Hum. Resour. Man. 2002, 6, 36-48.

23. Watad, M.; Ospina, S. Integrated Managerial Training: A Program for Strategic Management Development. Public. Pers. Manag. 1999, 28, 185.

24. Streufert, S.; Nogami, G.Y.; Swezey, R.W.; Pogash, R.M.; Piasecki, M.T. Computer Assisted Training of Complex Managerial Performance. Comp. Hum. Behav. 1988, 4, 77-88. 
25. Baldwin, T.T.; Magjuka, J.R. Organizational Training and Signals of Importance: Linking Pretraining Perceptions to Intentions to Transfer. Hum. Resour. Manag. Quar. 1991, 2, 25-36.

26. Longenecker, C.O. Maximizing Transfer of Learning from Management Education Programs: Best Practices for Retention and Application. Dev. Learning Org. 2004, 18, 4-6.

27. Baldwin, T.T.; Magjuka, J.R. Training as an Organizational Episode: Pretraining Influences on Trainee Motivation. In Improving Training Effectiveness in Work Organizations; Ford, J.K., Kozlowski, S.W.J., Kraiger, K., Salas, E., Teachout, M.S., Eds.; Lawrence Erlbaum Associates: Mahwah, NJ, USA, 1997; pp. 99-127.

28. Clarke, N. Job/work Environment Factors Influencing Training Transfer within a Human Service Agency: Some Indicative Support for Baldwin and Ford's Transfer Climate Construct. Int. J. Hum. Resour. Man. 2002, 6, 146-162.

29. McSherry, M.; Taylor, P. Supervisory Support for the Transfer of Team-Building Training. Int. J. Hum. Resour. Man. 1994, 5, 107-119.

30. Smith-Jentsch, K.A.; Salas, E.; Brannick, M.T. To Transfer or Not to Transfer? Investigating the Combined Effects of Trainee Characteristics, Team Leader Support, and Team Climate. J. Appl. Psychol. 2001, 86, 279-292.

31. Cohen, D.J. What Motivates Trainees? Train Dev. J. 1989, 44, 91-93.

32. Huczynski, A.A.; Lewis, J.W. An Empirical Study Into The Learning Transfer Process in Management Training. J. Manage. Stud. 1980, 17, 227-240.

33. Mezirow, J. Transformative Theory of Adult Learning. In In Defence of the Lifeworld: Critical Perspectives on Adult Learning; Welton, M., Ed.; State University of New York Press: New York, NY, USA, 1995; pp. 39-70.

34. Peters, L.H.; O’Connor, E.J.; Eulberg, J.R. Situational Constraints: Sources, Consequences, and Future Considerations. Res. Pers. Hum. Res. Man. 1985, 3, 79-114.

35. Brinkerhoff, R.O.; Montesino, M.U. Partnerships for Training Transfer: Lessons from a Corporate Study. Hum. Resour. Manage. Quar. 1995, 6, 263-274.

36. Gaudine, A.P.; Saks, A.M. A Longitudinal Quasi-Experiment on the Effects of Post-raining Transfer Interventions. Hum. Resour. Manag. Q. 2004, 15, 57-76.

37. Lim, D.H.; Morris, M.L. Influence of Trainee Characteristics, Instructional Satisfaction, and Organizational Climate on Perceived Learning and Training Transfer. Hum. Resour. Manag. Quar. 2006, 17, 85-115.

38. Clarke, N. The Impact of In-Service Training within Social Services. Brit. J. Soc. Work 2001, 31, 757-774.

39. Michalak, D.F. The Neglected Half of Training. Train Dev. J. 1981, 35, 22-28.

40. Webb, T.L.; Sheeran, P. Does changing behavioural intentions engender behaviour change? A meta-analysis of the experimental evidence. Psychol. Bull. 2006, 132, 249-268.

(C) 2014 by the authors; licensee MDPI, Basel, Switzerland. This article is an open access article distributed under the terms and conditions of the Creative Commons Attribution license (http://creativecommons.org/licenses/by/4.0/). 